\title{
Dynamiques des unités de production laitière dans le bassin d'approvisionnement de la ville de Ségou au Mali
}

\author{
G. Morin ${ }^{1}$ D. Coulibaly ${ }^{2}$ C. Corniaux ${ }^{3}$ \\ R. Poccard-Chappuis ${ }^{3}$ S.I. Sidibé ${ }^{2}$ C.H. Moulin ${ }^{1 *}$
}

Mots-clés

Bovin laitier - Production laitière Commerce - Famille - Structure sociale - Mali.

\begin{abstract}
Résumé
L'augmentation de la demande en lait frais, liée à la croissance urbaine, peut être une opportunité pour le développement de l'élevage bovin autour de la ville. Pour le bassin d'approvisionnement de la ville de Ségou (Mali), dominé par des circuits commerciaux informels, l'objectif de l'étude a été de comprendre les transformations des unités de production laitière. Des entretiens ont été conduits auprès de vingt chefs de famille pour retracer l'histoire de l'élevage bovin et de la gestion du lait dans leur concession, des années 1970 à 2005. Trois types d'unités laitières ont été distinguées : a) les agroéleveurs avec un faible prélèvement et une gestion du lait par les femmes, b) les agroéleveurs avec une production laitière soutenue et une gestion du lait par le chef de famille, et c) les commerçants urbains avec un troupeau bovin à orientation laitière. Le commerce du lait en ville a été un facteur de développement de la production laitière par extension du bassin d'approvisionnement (unité de type b), mais il n'a pas permis l'émergence de nouvelles techniques de production, notamment du fait du manque de crédit (types b et c) ou de la répartition des rôles entre hommes et femmes dans la gestion du lait (type a). Les éleveurs ont cependant fait évolué leurs pratiques : allotement des laitières, augmentation de la mobilité du reste du troupeau, complémentation en saison sèche, stockage des résidus de cultures, en réponse à la raréfaction des ressources à pâturer.
\end{abstract}

\section{INTRODUCTION}

Les systèmes de production laitiers évoluent sous l'influence de nombreuses contraintes ou opportunités locales. Le marché urbain a toujours été dans l'histoire un moteur important du développement des productions animales et plus particulièrement du lait (12, 13, 31). En Afrique de l'Ouest, en l'absence de chaîne du froid, les marchés de consommation urbains des villes moyennes sont des opportunités de développement de la production dans des bassins

1. SupAgro, UMR Errc, Montpellier, F-34060 France.

2. IER, Bamako, Mali.

3. Cirad, UR 18, Montpellier, F-34398 France.

* Auteur pour la correspondance

Montpellier SupAgro, UMR Errc, 2 place Pierre Viala, 34060 Montpellier Cedex 1, F-34060 France.

Tél. : +33 (0)4 99612365 ; fax : +33 (0)4 67545694

E-mail : moulinch@supagro.inra.fr d'approvisionnement périurbains (22). Certains de ces bassins, notamment au Sénégal et au Mali, se sont structurés avec l'apparition de minilaiteries (6), ce qui a entraîné des changements techniques et organisationnels importants dans les élevages bovins qui approvisionnent ces unités de transformation $(11,16)$. Dans d'autres villes, au contraire, le développement du commerce urbain de lait frais passe par l'apparition de collecteurs allant chercher du lait à vélo ou à moto. Cette structuration du commerce du lait reposant sur des circuits informels est-elle également favorable au développement de l'élevage bovin périurbain et entraîne-t-elle des modifications dans les techniques de production de lait : santé animale, amélioration génétique, alimentation ? L'objet de cette étude a été de caractériser les évolutions des systèmes de production laitiers autour de la ville de Ségou au Mali où le commerce du lait frais est assuré par des circuits informels. Il s'agit également d'identifier les facteurs déterminants de ces évolutions ainsi que les facteurs de blocage, afin d'analyser le rôle du commerce informel du lait dans l'évolution des techniques de production. 
Ségou est située sur la rive droite du fleuve Niger, à environ 200 $\mathrm{km}$ au nord-est de Bamako. La population de Ségou était estimée à 85000 habitants en 1998 lors du dernier recensement officiel ; elle serait de 100000 en 2007. A la différence du cas de Bamako, le milieu rural est à proximité de la ville, notamment sur la rive gauche du fleuve. Les précipitations annuelles s'élèvent à $600 \mathrm{~mm}$ en moyenne, avec une saison des pluies de quatre mois, de juin à octobre. Les cultures pluviales sont principalement le sorgho et le petit mil, et des légumineuses, niébé et arachide. Le maraîchage est pratiqué en bordure du fleuve. Des zones irriguées permettent la culture du riz. L'élevage bovin est une activité ancienne aux alentours de Ségou, surtout chez les Peuls, et reste avant tout la forme principale de capitalisation des revenus. La rive gauche et les îlots du fleuve Niger, ainsi que la région du Séno, abritent d'importants pâturages ; les résidus de culture constituent également une ressource alimentaire importante pour les bovins. A côté des villages à dominante bambara, de nombreux villages peuls ont été créés par des bergers peuls qui gardaient les troupeaux des Bambaras ou des citadins. Ces Peuls sédentarisés sont devenus des agroéleveurs. Il existe donc autour de Ségou un élevage bovin de type agropastoral diversifié, représentant un potentiel pour la production laitière.

La production laitière locale (1 000 à 2500 1/jour) assure en partie (10 à 25 p. 100) l'approvisionnement de la ville. Le lait est produit dans un rayon de $30 \mathrm{~km}$ autour de la ville sur la rive droite du fleuve. Les villages peuls du bord de la rive gauche participent également à cet approvisionnement (8). Il n'existe plus d'unités de transformation artisanales ou d'industrie laitière travaillant avec le lait local. Les expériences de minilaiteries, pourtant bien développées dans plusieurs villes moyennes du Mali, ont en effet échoué à Ségou (6). Le lait local est donc commercialisé au travers de circuits dits informels. Des revendeuses des villages proches transportent le lait à pied ; elles assurent aussi une transformation, en caillé et féné, et vendent directement aux consommateurs, à domicile ou au marché. Des collecteurs emportent le lait frais des éleveurs à vélo ou à moto, le long des axes routiers. Ils vendent le lait directement aux consommateurs ou approvisionnent des revendeuses urbaines.

Pour comprendre le rôle de ces circuits informels dans l'évolution des techniques de production du lait, les auteurs ont mené une étude privilégiant une approche dans " le temps long » par l'analyse des transformations des pratiques des éleveurs $(4,10,21)$. La méthode d'étude était donc exclusivement qualitative et reposait sur l'analyse des déclarations des éleveurs, obtenues par entretien auprès des chefs de famille. Elle a permis de dresser une typologie des unités de production de lait et d'identifier les évolutions perçues comme majeures pour les éleveurs au cours des deux dernières décennies. Les intérêts et les limites de cette méthode sont ensuite discutés avant de tenter une présentation des dynamiques à l'échelle du bassin de production et de dégager les différents facteurs d'évolution.

\section{- MATERIEL ET METHODES}

\section{Démarche d'étude}

Pour analyser l'influence du marché urbain du lait sur les dynamiques des élevages bovins, une étude a été faite sur les transformations des pratiques des familles commercialisant aujourd'hui du lait pour les consommateurs urbains. Les auteurs se sont intéressés, par enquête rétrospective, aux changements techniques dans la conduite des bovins, mais également aux pratiques de gestion concernant les dépenses d'entretien du troupeau. Ils ont également porté leur attention sur l'utilisation du lait trait : autoconsommation, rémunération en nature des bergers et commercialisation.
Enfin, ils ont cherché à connaître l'utilisation des recettes de la vente du lait. La démarche mise en œuvre a repris la méthode proposée par Moulin et coll. (24) pour analyser les transformations des pratiques d'éleveurs dans des petites régions françaises : territoire d'une coopérative laitière caprine dans les Cévennes (26), élevage ovin transhumant hivernant dans la plaine de la Crau (25). Cette démarche repose sur des entretiens compréhensifs et donc sur l'analyse des dires des éleveurs pour identifier quels sont les changements majeurs intervenus dans les façons de produire et de gérer le lait. Les données quantitatives sur les structures des systèmes de production (cheptel, main d'œuvre, surfaces cultivées) et les flux de lait sont donc des déclarations des éleveurs et doivent être considérées avec précaution : elles ne sont données qu'à titre indicatif. Les méthodes qualitatives fondées sur des entretiens sont en revanche très efficaces pour comprendre le raisonnement des éleveurs (9) en matière de production du lait et sa valorisation.

\section{Echantillonnage}

Une enquête par questionnaire fermé avait été réalisée en 2003 chez 60 éleveurs commercialisant du lait, répartis sur les différents axes d'approvisionnement en lait de la ville (8). A partir d'éléments structurels (surfaces cultivées, taille du cheptel), fonctionnels (disponible en aliments grossiers et en concentrés par unité de bétail tropical, pratiques sanitaires) et sur la commercialisation du lait, une stratification en cinq groupes a été réalisée. Pour la présente étude, un sous-échantillon de 20 éleveurs a été constitué, en tirant au hasard des éleveurs répartis par axe d'accès à la ville et par strate. Cette stratégie assurait que la diversité des situations possibles, en matière de localisation par rapport au marché et de systèmes d'élevage, était bien représentée dans l'échantillon. En revanche, à partir de cet échantillon, il n'a pas été possible d'évaluer le poids des différentes situations dans la population totale des élevages commercialisation du lait.

\section{Recueil et traitement des données}

Des entretiens ont été réalisés entre janvier et mars 2006 auprès des responsables du troupeau dans les 20 unités de production retenues. L'objectif a été d'écouter l'histoire de leur élevage bovin dans la concession, depuis la constitution du troupeau par la famille ou au moins depuis l'accession à la tête du troupeau du responsable actuel. Différents thèmes ont été abordés au cours de l'entretien : la famille et ses activités extra-agricoles ; le troupeau bovin, avec sa constitution, sa localisation saisonnière, l'alimentation et les pratiques sanitaires ; le lait, avec la traite, les débouchés, la gestion de la recette ; enfin, les cultures, en précisant l'utilisation des sousproduits pour l'alimentation animale. Deux entretiens, à raison de 1 h 30 à 2 h 30 par passage, ont été réalisés par deux enquêteurs, le premier relançant l'éleveur pour lui faire préciser des éléments de son récit, l'autre prenant des notes et vérifiant que tous les thèmes de la grille d'entretien étaient abordés. Au cours du premier entretien, l'objectif était d'écouter l'éleveur exposer la situation actuelle, puis de retracer l'histoire du troupeau, en insistant sur les changements survenus : période, justification, changements associés. Un compte-rendu de l'entretien était réalisé immédiatement après et une première représentation graphique de la chronique de l'unité de production était tracée afin de repérer les informations manquantes et les incohérences éventuelles. Le deuxième entretien avait pour objet de compléter et recouper les informations afin d'obtenir la chronique la plus fiable possible.

Suite aux entretiens, un premier traitement de l'information a été réalisé pour produire une chronique de l'unité de production sous forme d'un schéma synthétique (figure 1) et d'un texte. Cette chronique montre : i) la succession de périodes au cours desquelles 


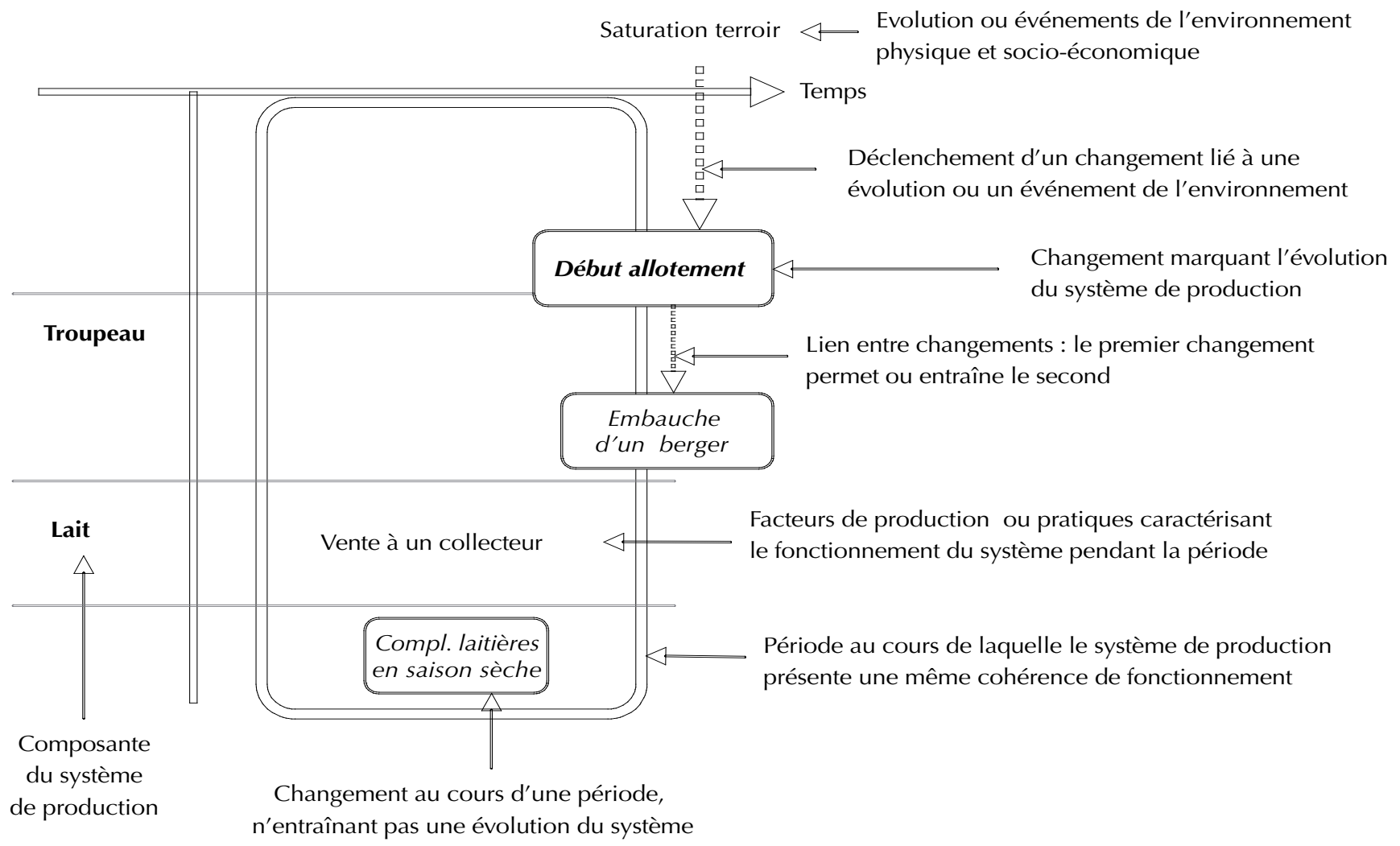

Figure 1 : éléments de représentation graphique de la chronique d'un système de production.

le système de production de la famille présentait une cohérence de fonctionnement (18), ce qui n'excluait pas des changements au cours de la période, comme par exemple l'augmentation des effectifs du troupeau ; ii) les changements survenus, dans la structure et le fonctionnement, notamment ceux qui signalaient le passage d'un système à un autre.

L'ensemble des vingt chroniques ont alors été analysées, tout d'abord en réalisant une typologie des unités de production, sur la base de leur situation actuelle en termes de conduite du troupeau, de l'utilisation du lait trait et de la gestion des recettes. Pour chaque type, les différents changements mis en œuvre par les unités de production du groupe ainsi que les éléments invariants ont été répertoriés. Si toutes les unités regroupées dans un type présent n'avaient pas suivi la même évolution, différentes trajectoires d'évolution étaient alors distinguées.

\section{RESULTATS}

\section{Diversité des unités de production de lait}

Trois types d'élevages commercialisant du lait pour approvisionner le marché urbain de Ségou ont été distingués. Leurs principales caractéristiques sont rassemblées dans le tableau I et la figure 2 présente leur localisation au sein du bassin d'approvisionnement.

\section{Type A : agroéleveurs avec faible prélèvement de lait et gestion du lait par les femmes}

Ce type regroupait des familles peules sédentarisées de longue date autour de Ségou. Elles pratiquaient des cultures : petit mil et sorgho, en partie en association avec du niébé, du riz, du maraîchage, des vergers et l'élevage de ruminants associant bovins et caprins. Le troupeau bovin était de 40 à 160 têtes et assurait de multiples fonctions, notamment la production de fumures organiques pour les cultures. Du fait de l'extension des surfaces cultivées, les surfaces pastorales étaient très réduites et ne permettaient pas le maintien du gros du troupeau dans le village où résidait la famille. Le troupeau ne restait sur le terroir villageois que pendant la période de vaine pâture, soit pendant deux à trois mois ; il était éloigné, durant la fin de la saison sèche, sur des îlots pour les villages proches du fleuve, par exemple, mais aussi durant la saison des cultures (transhumance proche ou lointaine). Quelques laitières, moins d'une dizaine, et leurs veaux, ainsi que les animaux trop faibles pour les déplacements et les bovins de traits au moment des travaux agricoles étaient gardés sur le territoire du village. Après la vaine pâture, ce lot d'animaux recevait des fourrages stockés (paille de brousse, éventuellement tiges de céréales). A partir de janvier, des concentrés étaient également distribués (son de céréales, tourteau ou graine de coton) en quantités limitées pour les vaches : une mesure de 1 à 2 1/jour (la mesure utilisée habituellement est la boite de concentré de tomate d'un litre). Les fanes de légumineuses n'étaient pas données aux bovins mais gardées pour les petits ruminants et les ânes ou bien vendues. Le chef de famille assurait l'entretien du troupeau (soins, sel, alimentation) grâce à des revenus propres : vente de fanes ou de foin de brousse, vente de taurillons, éventuellement bénéfices d'opérations d'embouche.

Les quantités de lait trait étaient faibles toute l'année : une dizaine de litres quotidiens, avec 0,5 à 2 litres par vache traite. La traite avait lieu deux fois par jour : le lait du soir était autoconsommé, le lait du matin était vendu par les femmes. Elles transportaient le lait à pied en ville pour le vendre à domicile. Les femmes géraient également la recette du lait : celle-ci devait tout d'abord couvrir les dépenses domestiques, les surplus servant aux dépenses personnelles des femmes.

Dans ces unités, le chef de famille dépensait pour se procurer des intrants, alors qu'il ne touchait pas la recette du lait. La conduite du troupeau était donc organisée de façon à assurer un prélèvement de lait suffisant pour couvrir l'autoconsommation, les dépenses 


\section{Tableau I}

Caractéristiques de production et de commercialisation des trois types d'unités laitières

\begin{tabular}{|c|c|c|c|}
\hline & $\begin{array}{c}\text { Type A } \\
\text { Agroéleveurs avec faible } \\
\text { prélèvement de lait et gestion } \\
\text { du lait par les femmes }\end{array}$ & $\begin{array}{c}\text { Type B } \\
\text { Agroéleveurs avec production } \\
\text { laitière soutenue et gestion } \\
\text { du lait par chef de famille }\end{array}$ & \begin{tabular}{l}
\multicolumn{1}{c}{ Type $\mathrm{C}$} \\
Commerçants urbains \\
avec troupeau bovin \\
à orientation laitière
\end{tabular} \\
\hline Main d'œuvre salariée pour l'élevage bovin & Aucune & Bergers & Bergers, manoeuvres \\
\hline Taille du troupeau & 40 à 110 têtes & 20 à 110 têtes & 50 à 130 têtes \\
\hline Allotement des laitières & 4 à 10 laitières & 7 à 10 laitières & 10 à 25 laitières \\
\hline Complémentation des laitières en SS & Faible & Moyenne $(2-4$ kg/VL/j) & Forte $(4-5 \mathrm{~kg} / \mathrm{VL} / \mathrm{j})$ \\
\hline Fourrages stockés pour alimentation bovins & $\begin{array}{l}\text { Foin de brousse } \\
\text { Tiges de céréales }\end{array}$ & $\begin{array}{l}\text { Tiges de céréales } \\
\text { Foin de brousse }\end{array}$ & $\begin{array}{l}\text { Tiges de céréales } \\
\text { Foin de brousse }\end{array}$ \\
\hline Quantités traites & $\begin{array}{l}\text { SP : } 10-15 \mathrm{l} / \mathrm{j} \\
\text { SS : } 5-10 \mathrm{l} / \mathrm{j}\end{array}$ & 10 à $40 \mathrm{l} / \mathrm{j}$ & 10 à $40 \mathrm{l} / \mathrm{j}$ \\
\hline Transport du lait en ville & Femmes de la famille & Collecteurs & $\begin{array}{l}\text { Main d'œuvre familiale } \\
\text { ou manœuvre salarié }\end{array}$ \\
\hline Commercialisation du lait en ville & $\begin{array}{l}\text { Femmes de la famille } \\
\text { Revendeuses }\end{array}$ & Collecteurs & $\begin{array}{l}\text { Femmes de la famille } \\
\text { Revendeuses }\end{array}$ \\
\hline Gestion de la recette de la vente du lait & Femmes & Chef de famille & Chef de famille \\
\hline Utilisation de la recette de la vente du lait & $\begin{array}{l}\text { Dép. domestiques } \\
\text { Dép. des femmes }\end{array}$ & $\begin{array}{l}\text { Dép. domestiques } \\
\text { Entretien du troupeau }\end{array}$ & Entretien du troupeau \\
\hline Revenu utilisé pour l'entretien du troupeau & $\begin{array}{c}\text { Vente fanes } \\
\text { Vente taurillons } \\
\text { Embouche bovine }\end{array}$ & $\begin{array}{l}\text { Vente fanes } \\
\text { Vente taurillons } \\
\text { Embouche } \\
\text { Vente du lait }\end{array}$ & $\begin{array}{l}\text { Commerce } \\
\text { Embouche } \\
\text { Vente du lait }\end{array}$ \\
\hline
\end{tabular}

VL : vache laitière ; SS : saison sèche ; SP : saison des pluies ; Dép : dépenses

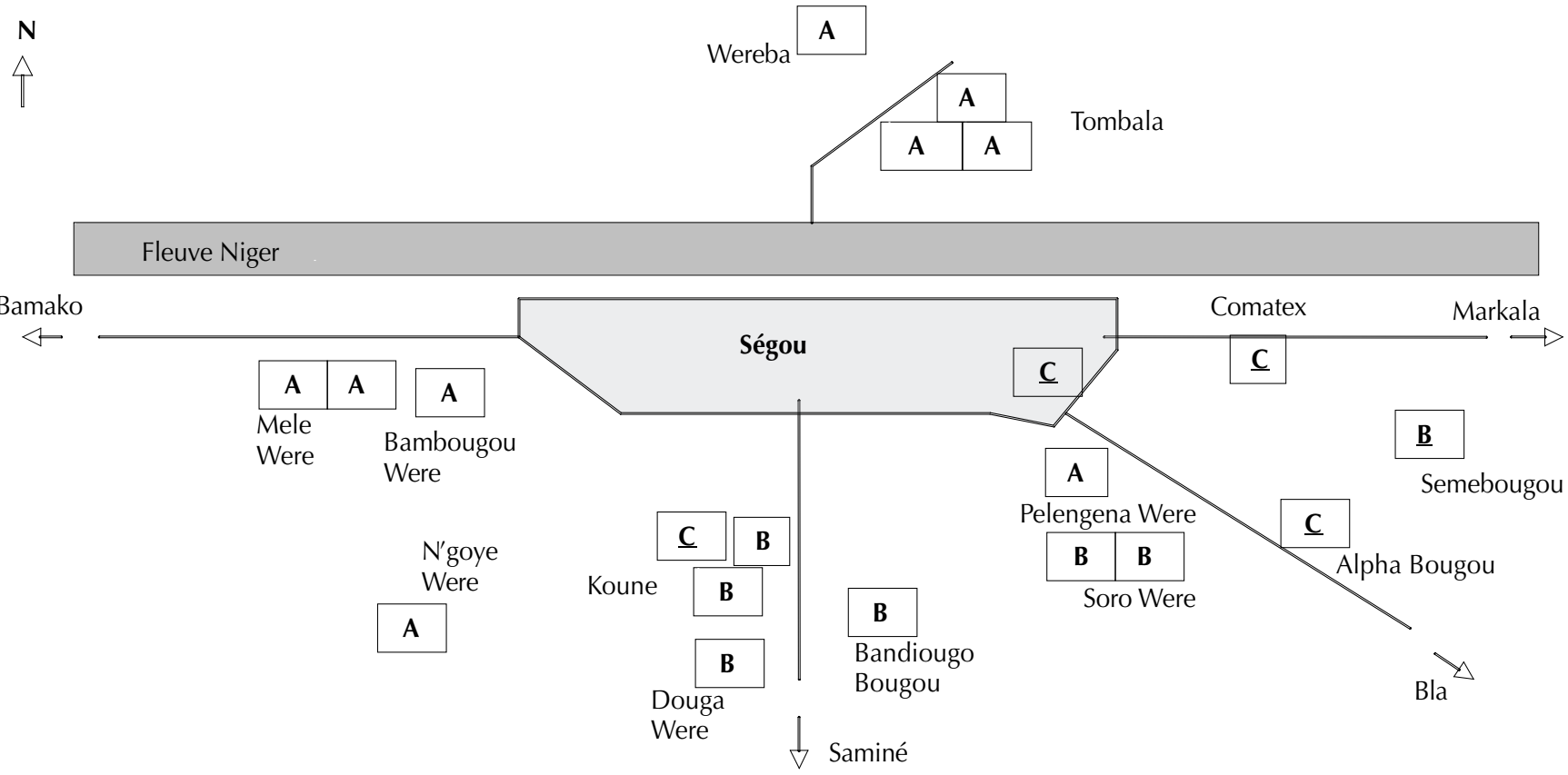
A : agroéleveurs avec faible prélèvement de lait et gestion du lait par les femmes
C : commerçants urbains avec troupeau bovin à orientation laitière
B : agroéleveurs avec production laitière soutenue et gestion du lait par le chef de famille
$\underline{\text { C } ~: ~ e ́ l e v e u r ~ r e ́ s i d a n t ~ e n ~ v i l l e ~}$ localisation du lot de laitières

Figure 2 : localisation des unités de production de lait enquêtées par types. 
domestiques et les dépenses des femmes. Le chef de famille ne cherchait pas à exploiter plus intensément le potentiel de production laitière du troupeau bovin : garder plus de laitières sur le village, distribuer plus d'aliment par vache n'avaient d'intérêt pour lui que s'il récupérait une partie de la recette du lait. Les dépenses en intrants alimentaires et vétérinaires étaient destinées surtout à garantir la pérennité du troupeau, patrimoine familial, et à assurer des recettes gérées par le chef de famille, notamment l'embouche, avec des animaux mieux soignés que les laitières : stabulation permanente, déparasitage systématique.

\section{Type B : agroéleveurs avec production laitière soutenue et gestion du lait par le chef de famille}

Ce type regroupait des familles peules et bambaras, pratiquant l'intégration agriculture-élevage, avec la traction animale, la production de fumier grâce au parcage d'une partie des animaux avec des pailles de céréales comme litière et l'utilisation des sous-produits agricoles pour l'alimentation animale. La conduite globale du troupeau était identique à celle du type précédent : transhumance de saison des pluies, voire de fin de saison sèche, allotement sur le village des laitières, animaux faibles et bovins de trait. En revanche, la production laitière était plus soutenue, avec une distribution de concentrés plus importante en saison sèche et des pratiques d'affourragement en vert des laitières en saison des pluies pour certaines familles.

Cette exploitation accrue du potentiel laitier était liée à un mode de gestion du lait différent. Si les femmes de la concession vendaient elles-mêmes le lait, elles reversaient une partie de la recette au chef de famille. Dans d'autres cas, le lait était directement vendu par le chef de famille à un collecteur urbain disposant d'un vélo ou d'une moto ou à des revendeuses du village qui allaient à Ségou. La recette du lait était toujours utilisée pour les dépenses domestiques, mais elle était suffisante pour contribuer aussi en partie aux frais d'entretien du troupeau.

\section{Type $C$ : commerçants urbains avec troupeau bovin à orientation laitière}

C'étaient des familles peules de commerçants (en bétail, aliments, tissus, sable...) qui vivaient en ville. Les activités agricoles et d'élevage, localisées en zone périurbaine plus ou moins éloignées, étaient assurées par des salariés et supervisées par un membre de la famille. La conduite du troupeau bovin suivait le même calendrier que dans les cas précédents, avec transhumance en saison des pluies, retour sur le terroir proche de la ville pour la vaine pâture et transhumance éventuelle de saison sèche chaude. En revanche, l'orientation laitière était très marquée, avec l'allotement de toutes les vaches en lactation pour les garder proche de la ville et un soutien marqué de la production laitière en saison sèche avec la distribution de concentrés. Deux éleveurs (sur les cinq qui composaient ce type dans l'échantillon) avaient également tenté l'insémination artificielle ou acheté des géniteurs améliorés (zébus Maures) pour la production laitière.

Dans ces élevages, le lait était géré par le chef de famille, installé en ville. Après la traite effectuée par des bergers salariés, le lait était transporté en ville par un manœuvre salarié ou un membre de la famille. Le chef de famille vendait alors le lait aux femmes de la famille qui effectuaient la revente ou à d'autres revendeuses. Dans le premier cas, les femmes de la famille achetaient aussi du lait à d'autres éleveurs. Elles transformaient jusqu'à 501 par jour en lait caillé mis en sachets plastiques sertis, ou degué avec du petit mil, ou boissons lactées parfumées par l'ajout de jus de fruits en poudre. Puis elles commercialisaient leurs produits auprès des consommateurs à partir de la concession en ville. La vente du lait frais par le chef de concession, combinée au produit de l'embouche assuraient l'entretien du troupeau. Quant aux femmes, leurs activités de revente et éventuellement de transformation leur permettaient de satisfaire à différentes dépenses personnelles (pour ellesmêmes ou pour leurs enfants).

La localisation des différents types d'élevage s'expliquait par la présence du fleuve et le mode de commercialisation du lait. Il y avait peu d'éleveurs sur la rive gauche du fleuve commercialisant du lait à Ségou, située sur la rive droite, du fait de la durée de la traversée : plus d'une heure en pirogue en comptant le temps d'attente. Seules des familles peules dans lesquelles les femmes allaient vendre le lait en ville (type A) étaient installées sur la rive gauche. Les éleveurs de type B commercialisaient leur lait par l'intermédiaire de collecteurs qui allaient chercher du lait à moto ou à vélo sur la rive droite du fleuve, sans avoir à traverser le fleuve en pirogue. Enfin, les commerçants urbains (type C) avaient également installé leur troupeau sur la rive droite afin de faciliter les transports de lait et d'aliments entre la ville et le lieu du parcage des laitières.

\section{Evolutions dans la production et la valorisation du lait}

Les chroniques par famille ont permis de remonter parfois jusqu'aux années 1970 et de saisir quelles ont été les évolutions : pérennité ou changements de certaines pratiques de production et de valorisation du lait. Ces chroniques ont été analysées pour chacun des trois types d'unités de production de lait. Cette analyse a cherché à mettre en évidence les déterminants des changements, d'après les raisons données par les éleveurs, notamment en faisant des liens entre changements de pratiques et événements survenus dans la famille ou dans son environnement.

\section{Type A : changements de conduite pour maintenir une production laitière gérée par les femmes}

Pour les familles peules de type A, l'élevage bovin et la commercialisation du lait étaient des pratiques anciennes (figure 3). La gestion du lait par les femmes, avec sa commercialisation et l'utilisation de la recette, était un invariant sur la période couverte par les chroniques (1970-2005). La vente du lait assurait toujours les dépenses domestiques et les dépenses des femmes ; l'entretien du troupeau était toujours à la charge du chef de famille. Le lait n'était donc pas un moyen privilégié pour augmenter les revenus de la famille, mais au cours des années, la production laitière a été préservée pour assurer les dépenses domestiques et les dépenses des femmes.

La mobilité du troupeau était ancienne, attestée dès les années 1970, ce qui nécessitait dès cette époque l'allotement de quelques laitières maintenues sur le village afin d'assurer la production de lait pour la famille sédentaire assurant les travaux de cultures. En revanche, les pratiques de conduite du troupeau et du lot des laitières ont évolué. Tout d'abord, la mobilité des troupeaux a augmenté, avec des transhumances de saison des pluies plus lointaines, en lien avec une diminution des surfaces de parcours proches en raison de l'extension des cultures et de l'urbanisation. La distribution d'aliments en complément du pâturage s'est également fortement développée (figure 4). Le stockage de fanes de légumineuses a démarré dans les années 1970, pour être vendues aux citadins qui pratiquaient l'embouche. Du foin de brousse était également stocké depuis les années 1970, pour la vente mais aussi pour la distribution aux animaux restant sur le village. Le contact avec les emboucheurs a incité les familles à distribuer des concentrés : son, graine de coton et tourteau, d'abord aux animaux faibles et malades (c'est le cas pour cinq familles sur huit pour la période 1980-84), puis aux vaches laitières gardées sur le village (sept éleveurs sur neuf pour la période 1985-90). A partir de 1990, toutes les familles ont 


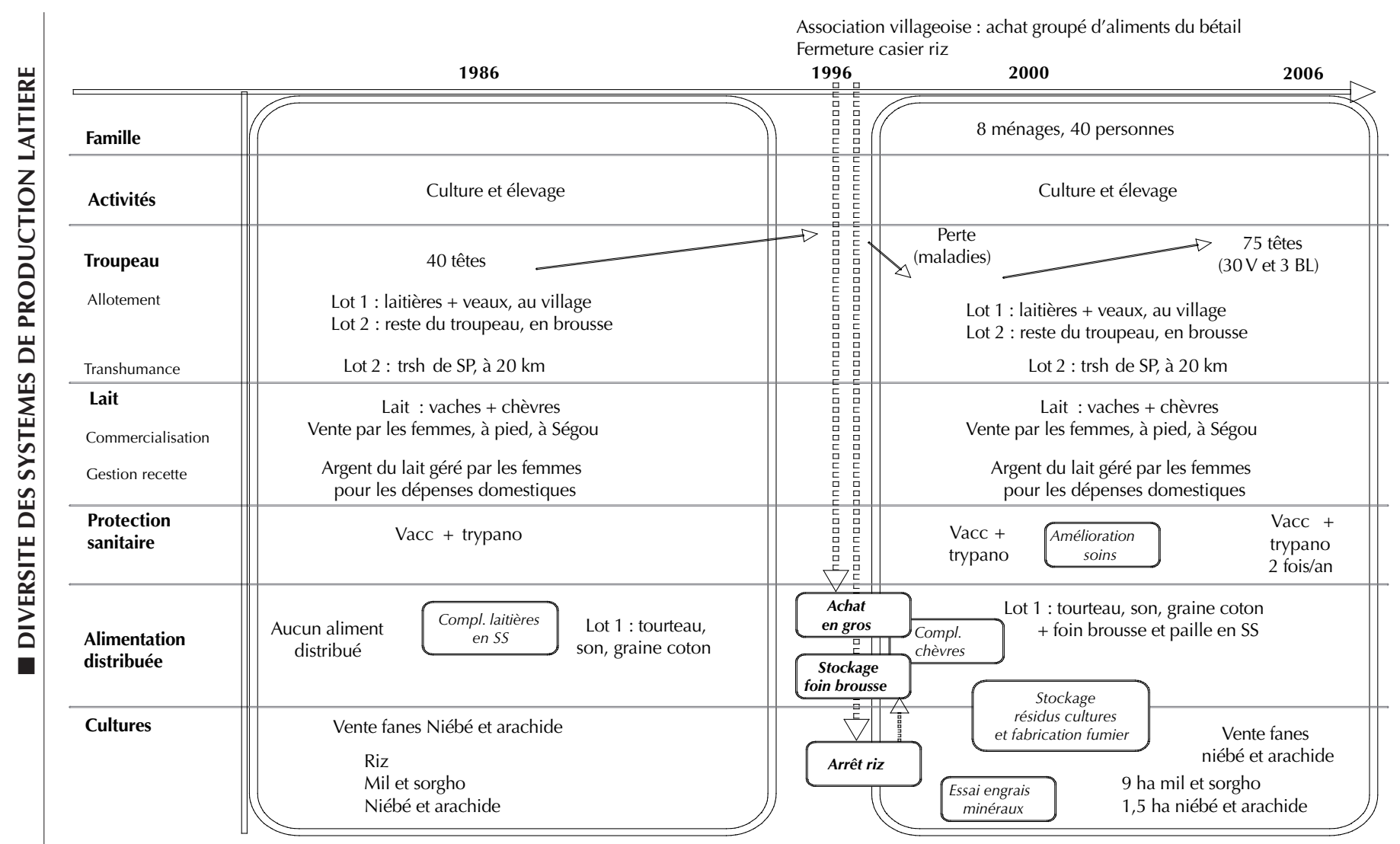

SS : saison sèche ; SP : saison des pluies ; V : vache ; BL : bœuf de labour ; Compl : complémentation ; trsh : transhumance ; Vacc : vaccination ; trypano : traitement préventif contre la trypanosomose.

Figure 3 : chronique d'une unité de production de type A (agroéleveur avec faible prélèvement de lait et gestion par les femmes).

10 Nombre d'unités laitières

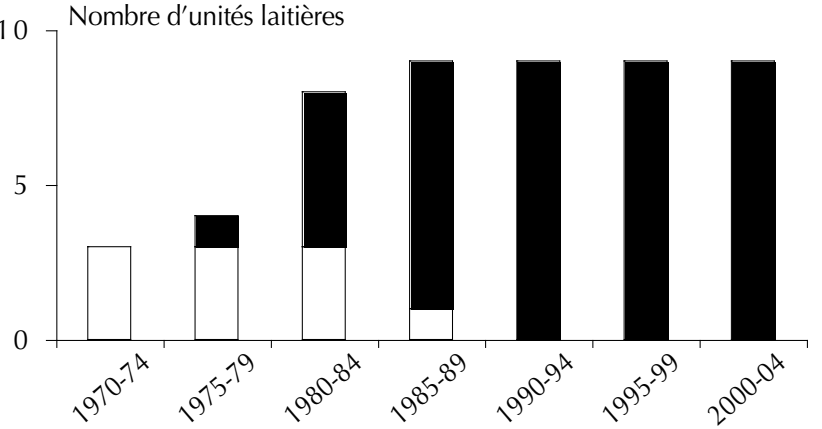

Complémentation des animaux faibles

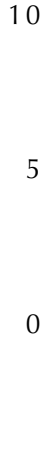

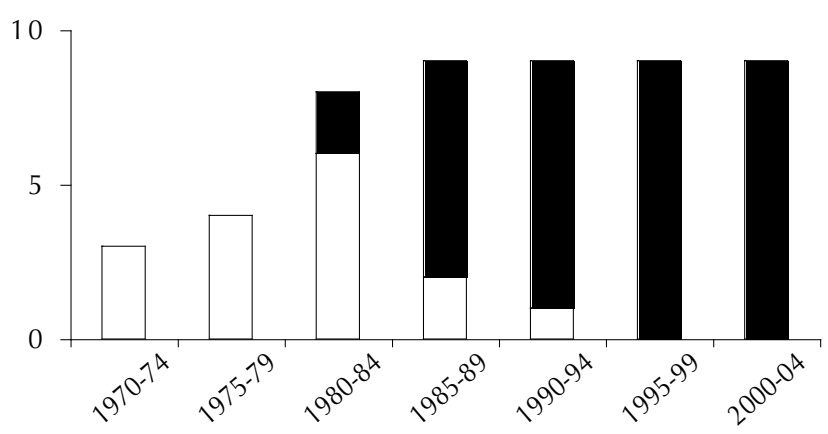

Complémentation des laitières en saison sèche

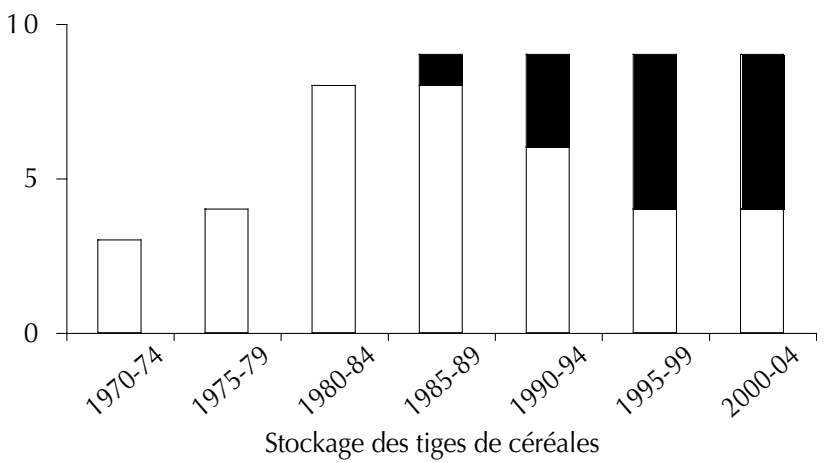

Non pratiqué

Unité laitière mettant en œuvre la pratique

Figure 4 : évolution entre 1970 et 2004 de quatre pratiques d'élevage pour les unités laitières du type A (agroéleveurs avec faible prélèvement de lait et gestion par les femmes). 
complémenté les animaux faibles et les laitières. Dans les années 1970, les tiges de céréales ont été laissées aux champs et utilisées en vaine pâture par les troupeaux. A partir des années 1990, une partie des familles ont récolté les tiges de céréales pour les distribuer aux animaux dans le parc de nuit. Les refus fournissaient une litière qui, mélangée aux déjections, permettait de produire du fumier. La fumure organique était alors de meilleure qualité que la «poudrette » (terre de parc) : c'était la raison principale de cette pratique, même si elle concourait également à augmenter la quantité de fourrages grossiers distribuée aux laitières.

\section{Type B : développement de l'élevage bovin et de la production laitière chez des agroéleveurs locaux}

Pour le type B, l'élevage bovin s'est développé à partir des années 1980, avec le démarrage de la production laitière et de sa commercialisation (figure 5). Les évolutions de la conduite technique des troupeaux ont été similaires à celles décrites pour les familles peules du type A (figure 6). En revanche, les recettes du lait ont augmenté, grâce à l'allotement d'un nombre plus grand de laitières (du fait de l'accroissement du cheptel) et à un soutien plus important de la production individuelle par la distribution de concentrés.

Dans ce groupe, la gestion du lait et de sa recette était le fait des hommes. Dans un premier cas, qui concernait notamment les familles bambaras, l'activité d'élevage bovin a été initiée par le chef de famille actuel. Lorsque la vente du lait a commencé, il a pris directement la gestion de sa recette, qu'il a utilisée pour couvrir les dépenses domestiques mais jamais celles privées des femmes. Dans un second cas, celui des familles peules, il y a eu passage de la gestion du lait, des femmes au chef de famille ; mais les chroniques réalisées n'ont pas permis de décrire ce passage. Les auteurs ont en effet observé deux types de configurations. Dans le premier, l'homme n'avait pas de troupeau lorsqu'il est devenu chef de famille. Pendant toute la période de constitution d'un troupeau, avant de pouvoir commercialiser du lait, les dépenses domestiques et les dépenses des femmes devaient être assurées par d'autres sources de revenu. Lorsque la commercialisation du lait a démarré, c'est l'homme qui en a pris la gestion. Dans le second, l'homme a initialement repris la gestion d'un troupeau de taille modeste (20-30 têtes) lorsqu'il est devenu chef de famille ; la gestion du lait était donc déjà passée aux hommes à la génération précédente, peut-être également à la faveur d'une rupture dans la commercialisation du lait. Ces ruptures dans la commercialisation du lait, qui favorisent la prise de gestion du lait par les hommes lorsque la vente de lait reprend, sont plus probables dans les familles où les liens entre ménages éclatent au moment du décès du chef de famille : certains ménages démarrent leur cycle de vie autonome sans pratiquement aucun capital initial sous forme de bovins. C'est également dans des familles de petite taille (un ou deux ménages) que les risques de perte de la quasi-totalité du cheptel sont les plus forts (suite à des épizooties, des sécheresses, des déstockages d'animaux pour faire face à des problèmes familiaux, etc.).

L'augmentation des quantités de lait vendues, rapportée par les éleveurs de ce groupe au cours des entretiens, pouvait avoir deux origines. Pour les uns, le bassin de commercialisation du lait restait identique. Les changements techniques, inspirés par les techniques d'embouche et rendus possibles par des revenus d'autres activités, ont permis une augmentation de la quantité de lait produit et ainsi de la recette du lait. Une fois payées les dépenses domestiques, les surplus de la recette du lait servaient à payer une partie de l'entretien du troupeau : achat d'aliments, soins vétérinaires. Pour les autres, au début de la commercialisation du lait, la vente se faisait sur le village, à destination donc de consommateurs ruraux. Avec le développement urbain, des collecteurs ont commencé à acheminer du lait de ces villages vers Ségou, en achetant le lait entre
150-175 FCFA/l en saison des pluies et entre 200-225 FCFA/l en saison sèche. Les éleveurs ont ainsi changé de débouchés et vendu leur lait un peu plus cher; de 25 à 50 FCFA de plus par litre, par rapport à la vente aux consommateurs ruraux. Ceci les a incité à augmenter la production laitière, avec les mêmes moyens techniques que dans le premier cas.

\section{Type $C$ : développement de l'élevage bovin et de la production laitière à partir des revenus du commerce}

Ces familles sont venues s'installer à Ségou dans les années 19601970 (figure 7). Originaires du Nord (Nioro, Mopti), elles étaient de tradition pastorale mais ont démarré des activités commerciales à Ségou, leurs animaux restant éventuellement confiés à des parents dans leurs villages d'origine. Les revenus commerciaux ont été capitalisés dans un nouveau cheptel bovin. Dans un premier temps, la recette du lait a été gérée selon le mode d'organisation traditionnel peul. La gestion du lait a changé lorsque les femmes, habitant en ville, sont devenues transformatrices et revendeuses. Elles achetaient alors le lait à leur époux. La recette du lait était utilisée par l'homme pour payer en partie les dépenses d'entretien du troupeau.

Sur le plan des techniques de production, les évolutions étaient comparables à ce qui se passait dans les autres types. Cependant, les activités commerciales dégageaient des revenus qui pouvaient être capitalisés sous forme de cheptel et permettaient de préfinancer les achats d'intrants pour entretenir et soutenir la production d'un nombre important de laitières. Ces revenus permettaient aussi de payer les salaires de bergers et manœuvres pour tous les transports (lait, aliments du bétail) nécessaires entre la ville et le lieu de parcage des laitières. Les revenus des autres activités et les relations sociales qu'entretenaient ces commerçants urbains leur ont également permis d'améliorer leur élevage bovin en introduisant des géniteurs améliorés (zébus Maures) ou en faisant appel à l'insémination artificielle (sur une ou deux vaches) avec des races laitières exotiques. La proportion d'animaux métis est restée cependant très faible.

\section{DISCUSSION}

\section{Intérêts et limites du recueil de données et de l'échantillonnage}

Cette étude, centrée sur les évolutions des unités de production, a relevé de la zootechnie-système. La démarche mise en œuvre a privilégié le recueil des récits des responsables de troupeau, en s'inspirant des méthodes des sciences sociales, notamment la technique d'entretien (19). Les informations issues de ces entretiens étaient de deux natures : des faits (taille du troupeau, date du démarrage de la vente de lait) et des représentations des éleveurs sur leur histoire, qui justifiaient leurs changements de pratiques en fonction des éléments qu'ils pensaient - a posteriori - avoir été les facteurs de leurs décisions : "J'ai commencé à vendre du lait en augmentant le prélèvement sur le troupeau quand un collecteur est venu chercher du lait au village »; "j'ai donné du son aux laitières en saison sèche pour avoir plus de lait... ». Les données quantitatives et le positionnement des événements dans le calendrier n'étaient que déclaratifs, mais le fait d'effectuer deux passages chez le même éleveur a permis de faire des recoupements qui ont assuré une bonne fiabilité des données. Sur la base de ces déclarations, le premier traitement a consisté à construire une représentation de la chronique. Celle-ci a combiné l'interprétation que faisait l'éleveur de sa propre histoire et celle qu'en a fait l'enquêteur. C'était ce dernier, par exemple, qui a réalisé le découpage de la chronique en plusieurs périodes, correspondant à une succession de systèmes de production. Ce découpage relevait de choix fait par l'observateur, 


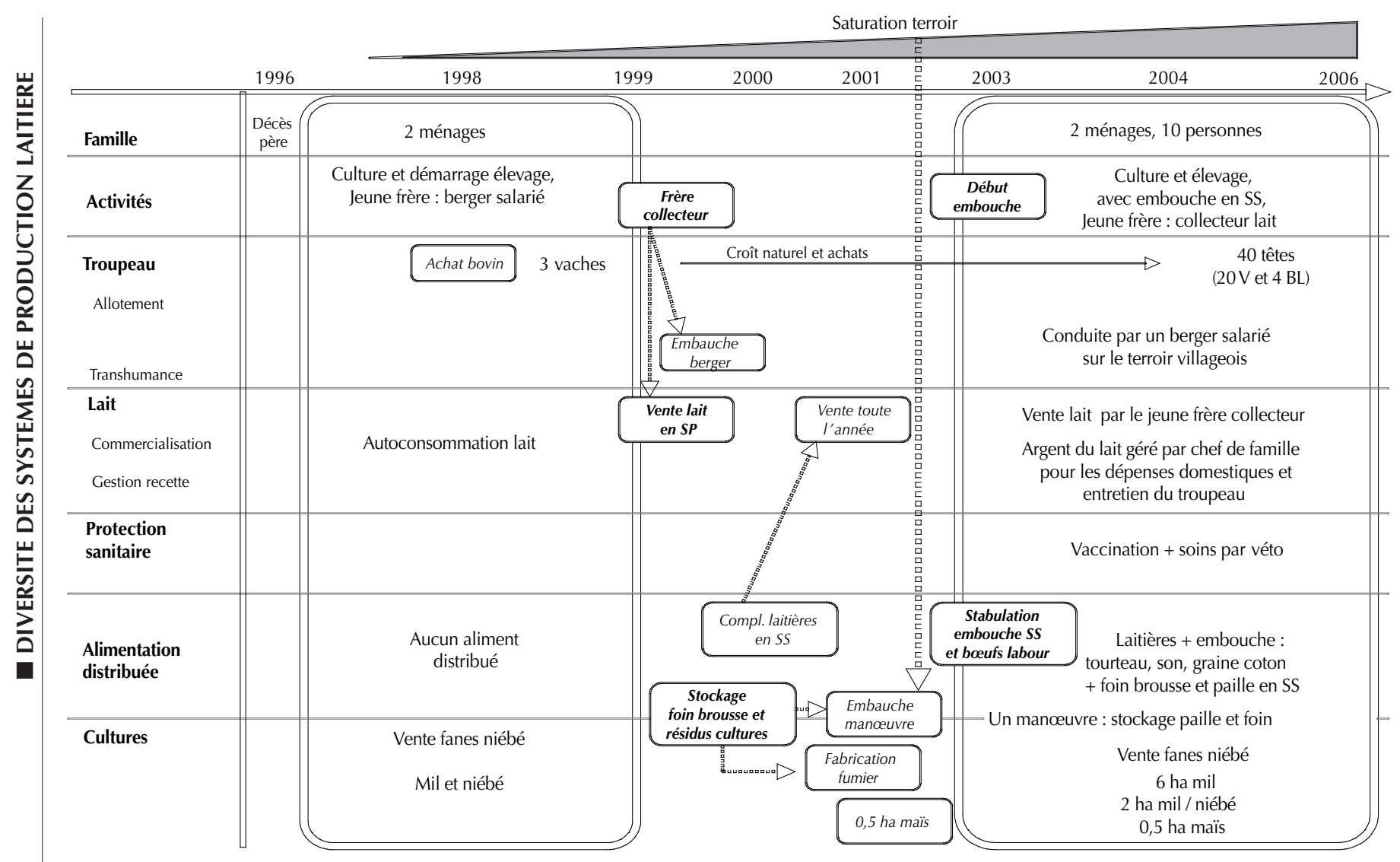

SS : saison sèche ; SP : saison des pluies ; V : vache ; BL : bœuf de labour ; Compl : complémentation.

Figure 5 : chronique d'une unité de production de type B (agroéleveur avec production laitière soutenue et gestion du lait par le chef de famille).

10 Nombre d'unités laitières

0

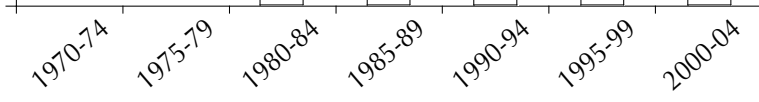

Complémentation des animaux faibles

10

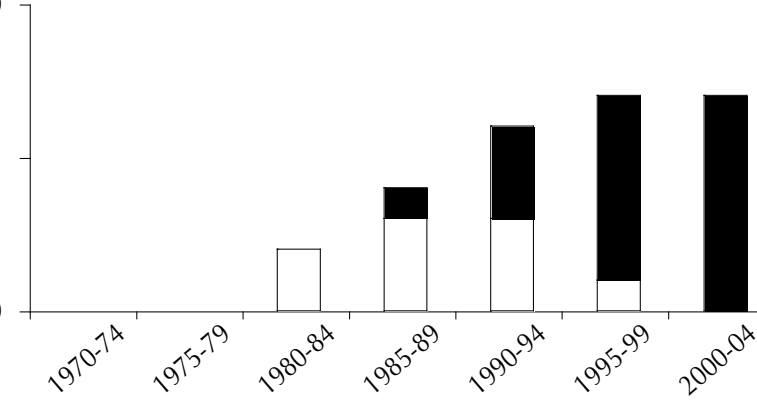

Stockage du foin de brousse

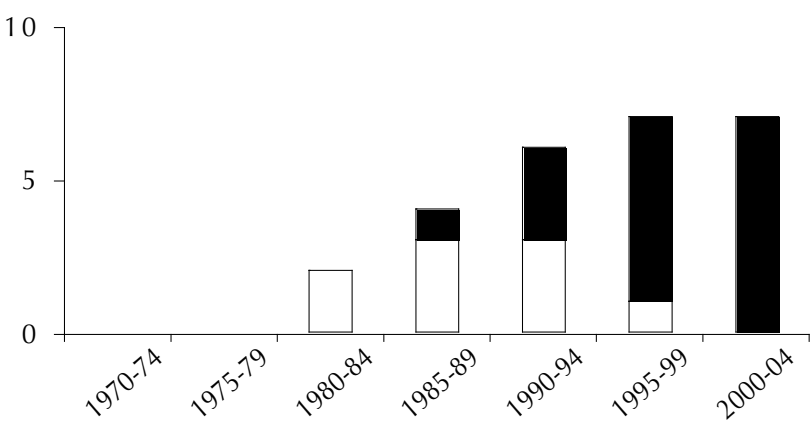

Complémentation des laitières en saison sèche

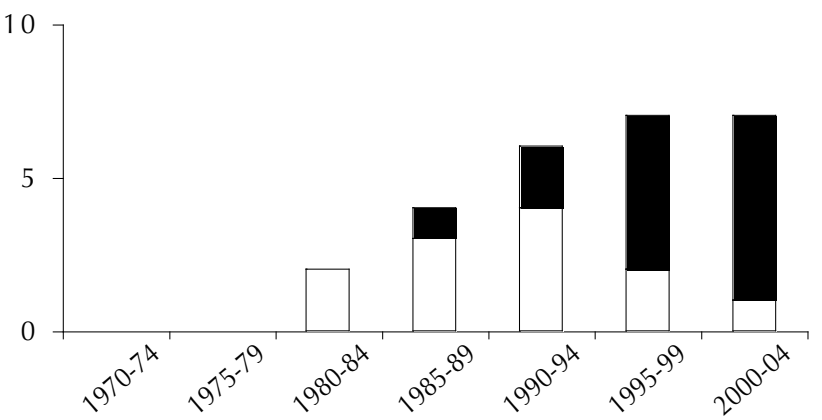

Stockage des tiges de céréales

Unité laitière mettant en œuvre la pratique

Figure 6 : évolution entre 1970 et 2004 de quatre pratiques d'élevage pour les unités laitières du type B (agroéleveur avec production laitière soutenue et gestion du lait par le chef de famille). 


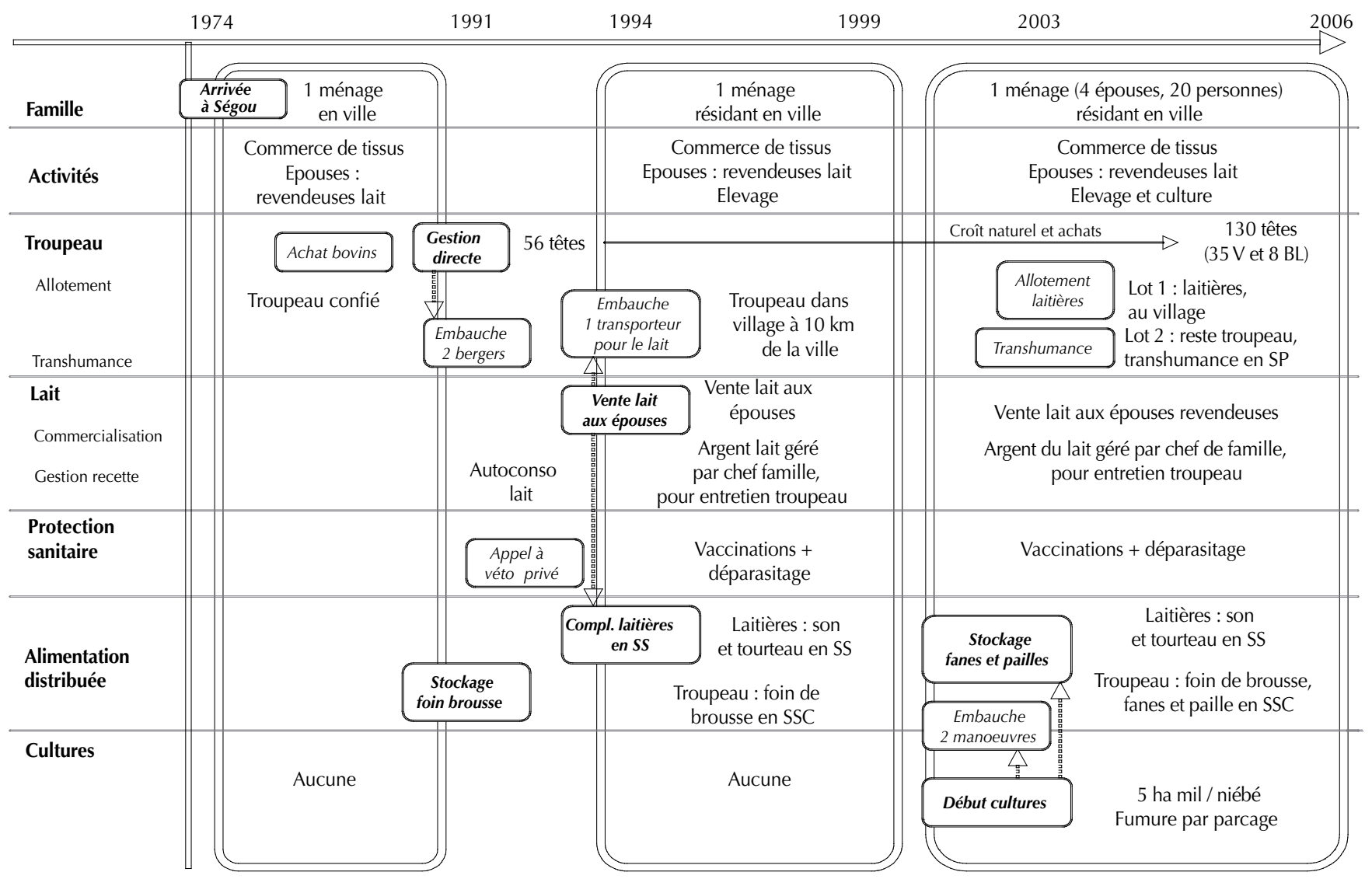

SS : saison sèche ; SSC : saison sèche chaude ; SP : saison des pluies ; V : vache ; BL : bœuf de labour ; Compl : complémentation.

Figure 7 : chronique d'une unité de production de type C (commerçants urbains avec troupeau à orientation laitière).

mais sur la base d'éléments objectifs qu'étaient les changements de pratiques relatés par l'éleveur. Ces chroniques, notamment sous leur forme synthétique graphique, ont constitué alors des données, rassemblées dans une base de cas, que d'autres personnes que l'enquêteur peuvent utiliser pour des analyses, comme il en est des résultats codifiés de la passation d'un questionnaire. Cet effort de formalisation est donc très important à plusieurs titres. Il permet tout d'abord de garantir la fiabilité des informations recueillies, grâce au premier traitement des informations entre les deux passages. Il permet aussi de prendre en compte, dans les travaux de terrain des zootechniciens, du temps long des transformations des systèmes agricoles (23). Cet aspect se révèle particulièrement précieux dans les contextes agraires où des changements importants sont intervenus, comme la croissance du marché urbain, la baisse des ressources naturelles, l'introduction de nouvelles techniques, dont il importe d'évaluer les impacts sur la production et les stratégies des éleveurs. Enfin, cette formalisation des résultats des entretiens permet leur transmission à de tierces personnes pour réaliser d'autres analyses.

L'intérêt de la méthode est d'aborder les dynamiques de la production dans un territoire, la prise en compte du temps long permettant de comprendre les stratégies réalisées par les collectifs gérant les troupeaux et la production laitière (17). Elle permet de comprendre les facteurs du maintien ou de développement de l'activité laitière dans une unité de production. En revanche, l'échantillonnage, réalisé sur la base d'éleveurs commercialisant aujourd'hui du lait pour le marché urbain, comporte un biais : aucune unité de production ayant arrêté de vendre du lait ne peut se retrouver dans l'étude. Or l'analyse des causes d'échec d'une activité au sein d'un système de production peut être très utile pour mieux comprendre les conditions de la réussite chez ceux qui aujourd'hui vendent du lait. Certains changements, ayant conduit à des impasses, peuvent donc échapper à l'analyse. Pour pallier cette difficulté, il faudrait élargir la base de l'échantillonnage en réalisant des entretiens chez des éleveurs qui ont arrêté le commerce du lait.

Les entretiens ont été réalisés auprès des chefs de famille, interlocuteurs obligatoires lorsqu'un enquêteur sollicite un entretien. Les auteurs n'ont donc appréhendé ici que le point de vue de ces chefs de famille : il serait utile de réaliser également des enquêtes auprès des autres membres de la famille, notamment des épouses, pour bien saisir tous les changements dans la gestion du lait. Mais la réalisation d'analyses fines auprès des différents membres de la famille nécessitent en général d'établir une relation suivie avec la famille (7).

\section{Dynamique et facteurs d'évolution du bassin laitier approvisionnant la ville de Ségou}

A partir de l'analyse des chroniques, il a été possible de tenter une reconstruction de la dynamique d'évolution du bassin laitier approvisionnant Ségou. Dans les années 1970, l'approvisionnement en lait local de la ville de Ségou était assuré par des familles peules sédentarisées sur la rive droite et la rive gauche du fleuve, à proximité de la ville. La production de lait se faisait uniquement au pâturage, avec une organisation de la mobilité du troupeau : transhumance de saison des pluies, ce qui nécessitait un allotement des laitières restant avec la famille ; troupeau restant proche de Ségou en saison sèche grâce à la proximité du fleuve, eau pour l'abreuvement, surfaces à pâturer sur des îlots, et les résidus de cultures. Cette organisation de la mobilité du gros du troupeau et de quelques bergers familiaux, associée à la sédentarité de la famille 
pratiquant l'agriculture était ancienne chez les Peuls du delta intérieur du Niger (14). Les femmes de ces familles peules allaient vendre le lait à pied en ville pour disposer d'une recette quotidienne permettant de couvrir les dépenses domestiques et leurs propres dépenses. Dans les villages plus éloignés du centre urbain, la production laitière des agroéleveurs bambaras ou peuls était faible : seuls quelques excédents étaient commercialisés sur le village, soit en vente directe aux consommateurs, soit par l'intermédiaire de revendeuses rurales.

A partir des années 1980, la croissance démographique de la zone a eu deux conséquences. Tout d'abord, un accroissement de la population urbaine de Ségou, avec une augmentation de la demande alimentaire, notamment en lait, puis une extension des cultures dans les villages proches de Ségou, pour répondre à l'accroissement de la population rurale. Ce dernier phénomène a entraîné une réduction des surfaces de pâturage en saison des pluies et une augmentation des disponibilités en sous-produits de culture pour l'alimentation animale et la production de fumure organique.

L'augmentation de la demande urbaine a entraîné une extension de l'aire d'approvisionnement de la ville en lait local. Dans les villages, à côté des revendeuses qui, à pied, ne pouvaient aller jusqu'en ville, de nouveaux opérateurs sont apparus : les collecteurs qui transportaient du lait à vélo ou à moto. Ce nouveau débouché pour les agroéleveurs qui n'accédaient pas jusque-là au marché urbain a été un moteur de l'accroissement de la production laitière chez certains d'entre eux, relevant du type B. De même en ville, l'accroissement de la demande en lait a permis à des femmes, épouses de commerçants du type $\mathrm{C}$, de s'installer comme revendeuses. Elles achetaient le lait à leur mari, ce qui changeait les rapports au sein de la famille, des échanges marchands s'instaurant entre époux, et s'approvisionnaient à d'autres sources. L'augmentation de la demande urbaine a donc entraîné un développement du commerce du lait, avec l'apparition de nouveaux opérateurs allant chercher du lait plus loin et un développement de la production laitière chez des agroéleveurs qui pratiquaient peu l'élevage bovin ou trayaient peu leurs vaches. Cette extension du bassin est restée cependant assez faible, car elle était limitée par les contraintes du transport artisanal du lait. Le même mécanisme a joué pour de nombreuses autres productions agricoles, ce qui a provoqué la diversification des sources de revenus des exploitations agricoles : fruits et légumes, fourrages pour l'élevage urbain, céréales.

Cependant, les changements techniques concernant la production laitière ont été peu importants : soutien de la production en saison sèche par des concentrés achetés, distribution de fourrages stockés, de qualité médiocre, avec des pailles de céréales (résidus de culture) ou du foin de brousse (produits de cueillette), les meilleurs fourrages, comme la fane de niébé, étant vendus sur le marché urbain. Ils n'ont également pas été très novateurs, ni par rapport aux évolutions des techniques de production du lait chez les Peuls plus ou moins sédentarisés d'Afrique de l'Ouest, ni par rapport à l'histoire de l'élevage laitier dans le monde. Les changements relatés au cours des chroniques n'étaient pourtant pas négligeables pour les familles, mais ils étaient essentiellement la réponse à une péjoration des conditions d'alimentation au pâturage, suite à la saturation des terroirs. Les techniques d'embouche étaient essentiellement à l'origine des ces pratiques de soutien de la production laitière. Celles-ci n'étaient pas une réponse au développement du commerce du lait. Plusieurs facteurs peuvent être avancés pour expliquer cette dynamique. Dans les hameaux peuls proches de la ville, la cohésion de grandes familles (jusqu'à 20 ménages, 80 personnes, avec une gestion commune d'un grand troupeau de la famille) permettait de garantir la pérennité du troupeau au cours des générations et le maintien de l'organisation entre hommes et femmes en ce qui concernait la gestion du lait. Les femmes tenaient le commerce du lait sur ces hameaux et il n'y avait pas de nouveaux opérateurs qui venaient le collecter. Le lait servait donc à couvrir les dépenses domestiques, c'est-à-dire l'essentiel du budget familial et les dépenses des femmes. Le gestionnaire du troupeau dépensait de quoi alimenter les vaches pour garantir le maintien de la production de lait nécessaire à la paix sociale entre membres de la famille, mais il ne cherchait pas à augmenter les recettes du lait. Sur le plan zootechnique, en effet, il n'avait pas intérêt à augmenter la traite, car il privilégiait l'alimentation du veau. Dans l'économie interne du ménage, augmenter les ventes signifiait, pour le chef de ménage, de donner davantage d'autonomie financière aux épouses et de perdre en partie le pouvoir (7). Dans les villages plus éloignés, les collecteurs venaient chercher du lait chez des agroéleveurs qui ont été incités à augmenter leur production, car ils assuraient eux-mêmes la gestion de la recette. Pour les familles peules, l'apparition, contraire à la tradition, d'une telle gestion de la recette par le chef de famille s'expliquait parce qu'il y a eu une rupture dans la vente de lait pendant une période plus ou moins longue, en raison de la perte provisoire du troupeau. Dans ces cas, l'homme, gérant les recettes du lait, avait intérêt à augmenter les quantités vendues. Mais l'augmentation est restée limitée. En effet, les collecteurs n'assuraient que la fonction de transport et de commercialisation du lait. Ils n'avaient pas les moyens de faire du crédit aux éleveurs pour leur permettre de développer de façon plus importante la production laitière. En l'absence de trésorerie et de système bancaire faisant du crédit aux agroéleveurs, les achats d'aliments étaient limités ; les cultures céréalières vivrières restaient une priorité pour les familles et empêchaient le développement de cultures fourragères. Chez les commerçants peuls (type C), les autres activités, notamment commerciales, permettaient de disposer d'un peu de crédit pour l'activité bovine ; ces éleveurs étaient également insérés dans des associations d'emboucheurs ou de commerçants en bétail qui leur donnaient accès à des aliments à des prix de gros ou même à des crédits pour ces achats. Si ceci leur permettait d'avoir les productions laitières les plus importantes des trois groupes, celles-ci restaient tout de même relativement faibles (tableau I). Les échecs des opérateurs de l'aval (type minilaiterie), qui auraient pu à la fois diffuser des messages sur les techniques d'amélioration de la production et fournir du crédit pour financer ces techniques, expliquaient en partie ce faible développement de la production laitière autour de Ségou. Le développement des grands bassins laitiers dans le monde s'est en effet réalisé avec l'appui de l'industrie laitière en aval $(3,30)$, les unités artisanales type minilaiterie jouant également ce rôle, à une moindre échelle, dans les villes moyennes d'Afrique de l'Ouest (6). Ces échecs pouvaient s'expliquer par la concurrence entre les différents types de produits laitiers offerts aux consommateurs urbains (à partir de lait local ou de poudre importée), par des difficultés de gestion de ces unités de transformation, mais aussi par des coûts d'opportunité en zone périurbaine plus favorables à d'autres activités agricoles que la production laitière. Cependant, cette étude, centrée sur les producteurs laitiers, ne permet pas d'aborder ces facteurs d'explication.

Dans les années 1990, deux événements majeurs ont pu marquer l'évolution des systèmes laitiers locaux : la dévaluation du franc de la Communauté financière africaine en 1994 et l'implantation d'une unité artisanale de transformation (Ségou-Lait) en 1996. Pour les économistes initiateurs de la dévaluation, il s'agissait de rendre les produits locaux plus compétitifs face aux importations : le lait était concerné, en raison de l'importance des importations de la poudre de lait importée, principal produit laitier consommé dans les villes d'Afrique de l'Ouest. Dans les chroniques, aucun impact de la dévaluation sur la production n'est apparu. Ceci montre le manque de réactivité de la filière locale face à des opportunités d'ouverture ou d'élargissement de marchés urbains. L'unité de transformation Ségou-Lait (1996-2003), d'une capacité de 
1000 l/jour, a très peu contractualisé avec les producteurs locaux, ne pouvant pas leur proposer de prix attractif par rapport à celui permis par la vente directe ou le circuit informel ; dès lors elle s'est essentiellement fournie en lait en poudre.

Au terme de cette étude, il est possible de tracer des perspectives de développement de la production laitière selon les types. Les familles de type A ne constituent pas un réservoir important d'augmentation de la production de lait, tant que la division entre hommes et femmes dans la gestion de la production laitière et l'utilisation du revenu du lait ne change pas. En revanche, la production laitière dans ce groupe est un moyen essentiel de régulation des rapports économiques entre hommes et femmes. Pour les familles de type $\mathrm{B}$, le potentiel de développement de la production est plus important, mais le faible niveau de capitalisation et d'offre de crédit limite les possibilités de développement de la production des unités existantes : l'augmentation de la production ne peut se faire alors que par extension du bassin d'approvisionnement, voire par sa délocalisation hors de la couronne périurbaine ; cette extension spatiale est limitée par les contraintes de transport (30). Dans le contexte actuel, le marché du lait est plutôt une opportunité pour les familles qui ont déjà un capital suffisant pour investir dans cette activité et financer la trésorerie nécessaire pour acheter les intrants au meilleur prix (type $\mathrm{C}$ ). La production laitière ne se révèle pas être ici un moyen décisif de lutte contre la pauvreté pour des petits éleveurs, contrairement à ce qui a été rapporté souvent ailleurs (1, 11, 28, 29). Le marché urbain, dans le contexte de circuits de collecte informels, ne se révèle pas non plus être un moteur efficace pour l'innovation dans les systèmes d'élevage laitier.

\section{Enseignements pour la zootechnie-système}

Cette étude montre bien l'importance de la prise en compte des modes de gestion du lait entre les différents membres de la famille. En termes de zootechnie-système, il est donc impossible d'aborder ces élevages en les considérant comme des systèmes pilotés par un acteur-décideur unique, comme cela a déjà été montré par des travaux en Afrique de l'Ouest (15) et en particulier en élevage bovin $(7,20)$. Pour comprendre les choix techniques des gestionnaires de la production et identifier les éléments qui limitent les possibilités de changements, il est nécessaire de suivre l'ensemble du processus qui met en jeu le lait, depuis la reproduction (qui commande le démarrage d'une lactation), jusqu'au prélèvement par la traite (domaine classique du zootechnicien), mais aussi jusqu'à la répartition et l'utilisation de la recette du lait (qui peut retourner ou non dans l'activité par la couverture d'une partie des achat d'aliments, de produits vétérinaires). Les pratiques de gestion $(2,5)$ font donc bien partie du champ des objets de la zootechnie-système. La typologie construite ici, outil pour comprendre les évolutions de la production laitière chez les éleveurs et dresser des perspectives d'avenir, repose autant sur les éléments de fonctionnement technique de la production que sur les pratiques de valorisation du lait et de gestion de la recette du lait.

L'analyse dans le temps long se révèle ici primordiale pour tenter de comprendre les conditions de passage d'une forme de rapport à une autre dans la gestion du lait et de sa recette : dans des familles peules du type $\mathrm{B}$, l'interruption de la vente de lait, consécutive à une quasi-absence de bovins dans la famille, permet d'expliquer un passage de la gestion des femmes aux hommes. Cette interruption n'est cependant pas nécessaire : dans des familles peules de Casamance (Sénégal), la stabulation de vaches en saison sèche pour la production de lait est effectuée par les hommes qui gèrent les recettes du lait vendu à une minilaiterie, alors que les femmes continuent à exploiter et à commercialiser la production laitière du troupeau extensif $(11,16)$.
Au-delà des rapports entre les différents membres de l'unité de production autour des flux physiques et monétaires générés par l'activité, l'organisation des réseaux d'acteurs qui interviennent dans le commerce d'un produit d'élevage est également un élément structurant des transformations des pratiques des éleveurs. Le changement d'intermédiaires - comme les collecteurs pour le marché urbain au lieu des revendeuses en milieu rural - et le développement de la production qui l'accompagne chez des agroéleveurs en sont un exemple. L'organisation spatiale et la contractualisation de ces réseaux sont particulièrement importantes pour comprendre les dynamiques à l'œuvre sur le terrain. Ici l'étude réalisée montre ses limites. Pour aller plus loin sur ces questions, il aurait fallu coupler l'approche par les producteurs avec une étude menée auprès des acteurs de la commercialisation du lait. Il aurait également fallu développer une étude des évolutions spatiales des territoires, support de l'activité d'élevage (27). Ceci aurait permis de mieux saisir les enjeux de la mise en relation d'espaces plus ou moins éloignés autour de la production et de la commercialisation du lait : localisation des vaches en lactation produisant le lait, mais également des troupeaux de plus en plus éloignés du centre urbain et qui assurent le remplacement de ces vaches en lactation.

\section{CONCLUSION}

Le commerce du lait local pour les consommateurs urbains de Ségou a été un facteur de développement de la production laitière par extension du bassin d'approvisionnement de la ville. Cette extension a été permise par les collecteurs transportant le lait à vélo ou à moto, et par la vente de lait par des agroéleveurs qui ont accru leur cheptel bovin et augmenté le prélèvement de lait sur leurs vaches. Cependant, ce commerce de lait, organisé autour d'une filière de vente directe, n'a pas permis l'implantation d'intermédiaires (transformation, distribution) qui auraient pu soutenir le développement de la production. Les éleveurs ont cependant fait évoluer la conduite de leurs troupeaux, en réponse à la raréfaction des ressources à pâturer. Le commerce du lait est peu intervenu dans ces changements, même si une partie des recettes du lait a été utilisée par certains éleveurs pour financer l'achat d'intrants pour la production.

En matière de développement de la production laitière, trois pistes de réflexion peuvent être dégagées de cette étude. Sur le plan des techniques de production, dans le cadre des conduites actuelles, des améliorations pourraient porter sur les pratiques de distribution d'aliments complémentaires, à la fois sur les fourrages distribués (amélioration de la valeur alimentaire des fourrages utilisés) et les concentrés (rechercher une meilleure complémentarité avec les fourrages). Au-delà des questions techniques et économiques (choix pour certains fourrages entre la vente ou leur transformation en lait et en viande par le troupeau) et de la diffusion de bonnes pratiques de complémentation, c'est aussi les circuits d'approvisionnement en aliments complémentaires qui doivent être organisés. Sur le plan de l'implantation d'une nouvelle unité de transformation artisanale voulant collecter du lait local, l'organisation du réseau d'acteurs acheminant le lait sur la ville est à prendre en compte tout autant que la présence d'un tissu d'éleveurs et de troupeaux bovins. Les relations sociales entre ces acteurs (revendeuses, collecteurs) et les producteurs de lait sont souvent intenses : c'est le cas des revendeuses dont les époux sont les principaux fournisseurs, mais également des collecteurs, qui peuvent aussi avoir des liens familiaux avec les gestionnaires de troupeaux. Ces liens peuvent expliquer les difficultés pour un nouvel opérateur de faire sa place sur certains axes d'acheminement du lait, même dans le cadre d'un marché en expansion. Pour concurrencer ces acteurs informels, l'offre de prix et de services associés 
proposés par un nouvel opérateur doit être suffisamment attractive pour inciter les éleveurs à changer de partenaires commerciaux (cas de Nestlé à Dahra). Enfin, sur le plan des politiques publiques, pour favoriser le développement de l'activité laitière, l'accès aux crédits pour les éleveurs semble être une priorité et permettrait une augmentation de la production chez les agroéleveurs du type B.

Ces enseignements tirés du cas particulier du bassin d'approvisionnement de la ville de Ségou ont sûrement une valeur plus générique, pour les situations dans lesquelles le bassin de production et le commerce de lait local est aux mains d'acteurs possédant peu de moyens (revendeuses, collecteurs). Les travaux en cours au Mali sur d'autres villes moyennes, comme Sikasso en zone cotonnière, devraient permettre de comparer la dynamique de différents bassins et de conforter ces analyses. Au-delà de cette approche qualitative mettant en évidence les grandes évolutions et les stratégies des différentes catégories de producteurs de lait, des études technico-économiques plus fines sont également nécessaires. La quantification des flux (matière, argent) sur au moins une campagne annuelle est indispensable, pour mieux évaluer l'intérêt économique de la production laitière pour les agroéleveurs.

\section{BIBLIOGRAPHIE}

1. BEBE B.O., UDO H.M.J, ROWLANDS G.J., THORPE W., 2003. Smallholder dairy systems in the Kenya highlands: breed preferences and breeding practices. Livest. Prod. Sci., 82: 117-127.

2. BROSSIER J., CHIA E., MARSHALL E., PETIT M., 2003. Gestion de I'exploitation agricole familiale. Dijon, France, Educagri, 215 p.

3. CALVEZ E., 2006. La filière laitière de I'ouest de la France : entre restructuration et mondialisation (1984-2004). In : Madeline P., Moriceau J.M., éds, Acteurs et espaces de l'élevage (XVII $-\mathrm{XXI}$ siècle). France, Rennes, Presses universitaires, p. 263-276. (Histoire rurale, $n^{\circ}$ 9)

4. CARON P., HUBERT B., 2000. De l'analyse des pratiques à la construction d'un modèle d'évolution des systèmes d'élevage application à la région Nordeste du Brésil. Revue Elev. Méd. vét. Pays trop., $\mathbf{5 3}: \mathbf{3 7 - 5 3}$

5. CHIA E., 1992. Une "recherche-clinique ": proposition méthodologique pour I'analyse des pratiques de trésorerie des agriculteurs (étude de cas en Lorraine). Paris, France, Inra. (Etudes et recherches sur les systèmes agraires et le développement $\mathrm{n}^{\circ} 26$ )

6. CORNIAUX C., DUTEURTRE G., DIEYE P.N., POCCARD-CHAPUIS R., 2005. Les minilaiteries comme modèle d'organisation des filières laitières en Afrique de l'Ouest : succès et limites. Revue Elev. Méd. vét. Pays trop., $58: 237-243$.

7. CORNIAUX C., VATIN F., FAYE B., 2008. Prise de décision en matière de production laitière au sein des concessions sahéliennes. Cah. Agric. (sous presse)

8. COULIBALY D., POCCARD-CHAPUIS R., KASSAMBARA I. CORNIAUX C., NIANG M., 2007. Rapport de recherche de la campagne 2005-2006 du projet Recherche de modes de gestion du troupeau pour une exploitation économique et durable des bovins laitiers dans les zones périurbaines du Mali. Montpellier, France, Cirad, $19 \mathrm{p}$.

9. DARRE J.P., HUBERT B., LANDAIS E., LASSEUR J., 1993. Raisons et pratiques. Dialogue avec un éleveur ovin. Etud. rurales, 131-132 : 153-163.

10. DEDIEU B., CHIA E., LECLERC B., MOULIN C.H., TICHIT M., 2008. L'élevage en mouvement. Flexibilité et adaptation des exploitations d'herbivores. Paris, France, Quae, 296 p.

11. DIEYE P.N., FAYE A., SEYDI M., CISSE S.A., 2002. Production laitière périurbaine et amélioration des revenus des petits producteurs en milieu rural au Sénégal. Cah. Agric., 11 : 251-257.

12. FANICA O., 2006. Du lait pour la capitale. La production laitière autour de Paris (1700-1914). In : Madeline P., Moriceau J.M., éds, Acteurs et espaces de l'élevage (XVII $-X X^{e}$ siècle). France, Rennes, Presses universitaires, p. 141-154. (Histoire rurale, $n^{\circ}$ 9)
13. FAO, 1996. Appui à la formation d'un programme national de promotion de la production laitière au Mali. Rapport de synthèse provisoire. Bamako, Mali, FAO, $159 \mathrm{p}$.

14. GALLAIS J., 1984. Hommes du Sahel. Paris, France, Flammarion, 289 p. (coll. Géographes)

15. GASTELLU J.M., 1980. Mais où sont donc ces unités économiques que nos amis cherchent tant en Afrique? Cah. Orstom Sér. Sci. Hum., 23 : 421-437.

16. GAULIER A., 2005. Etudes des innovations dans les ceintures laitières périurbaines en Haute Casamance. Thèse Master Sci. Développement agricole tropical, Cnearc, Montpellier, France, 122 p.

17. GIRARD N., 1995. Modéliser une représentation d'experts dans le champ de la gestion de l'exploitation agricole. Stratégies d'alimentation au pâturage des troupeaux ovins allaitants en région méditerranéenne. Thèse Doct., université Claude Bernard, Lyon I, France, 234 p.

18. GRAS R., BENOIT M., DEFFONTAINES J.P., DURU M., LAFARGE M., LANGLET A., OSTY P.L., 1989. Le fait technique en agronomie. Activité agricole, concepts et méthodes d'étude. Paris, France, L'Harmattan, 184 p.

19. KAUFMANN J.C., 1996. L'entretien compréhensif. Paris, France, Nathan, $126 \mathrm{p}$.

20. LHOSTE P., 1986. L'association agriculture-élevage. Evolution du système agro-pastoral au Sine-Saloum (Sénégal). Thèse Doct. Ing., INA Paris-Grignon, France, 314 p.

21. MADELRIEUX S., DEDIEU B., DOBREMEZ L., 2002. Modifications de I'utilisation du territoire lorsque les éleveurs cherchent à résoudre leurs problèmes de travail. Fourrages, $172:$ 355-368.

22. MEZTGER R., CENTRES J.M., THOMAS L., LAMBERT I.C. 1995. L'approvisionnement des villes africaines en lait et en produits laitiers. Rome, Italie, FAO, 105 p. (Production et santé animales $n^{\circ} 124$ ).

23. MOULIN C.H., COULIBALY D., POCCARD-CHAPUIS R., CORNIAUX C., 2005. Guide méthodologique pour l'analyse des changements dans les unités de production commercialisant du lait en zone urbaine au Mali. Montpellier, France, SupAgro, UMR Errc, 30 p.

24. MOULIN C.H., INGRAND S., LASSEUR J., MADELRIEUX S., NAPOLEONE M., PLUVINAGE J., THENARD V., 2008. Comprendre et analyser les changements d'organisation et de conduite de l'élevage dans un ensemble d'exploitations : propositions méthodologiques. In : Dedieu B., Leclerc B., Chia E., Moulin C.H., Tichit M., éds, L'élevage en mouvement. Flexibilité et adaptation des exploitations d'herbivores. Paris, France, Quae.

25. MOULIN C.H., PLUVINAGE J., BOCQUIER F., 2004. Les relations entre agrandissement des troupeau et changements de conduite : exemple des élevages ovins allaitant en Crau. Rencontres Rech. Ruminants, 11 : $145-148$

26. NAPOLEONE M., BOUTONNET J.P., 2004. AOC Pélardon : du compromis vers l'émergence d'actions collectives. Dynamiques de systèmes de production et des stratégies de commercialisation. In : Séminaire Sfer, les systèmes de production agricoles, performances, évolutions, perspectives, Lille, France, 18-19 nov. 2004, 11 p.

27. SABOURIN E., CARON P., TONNEAU J.P., 2004. Dynamique territoriales et trajectoires de développement local : retour d'expériences dans le Nordeste brésilien. Cah. Agric., 13 : 539-545.

28. SHAMSUDDIN M., GOODGER W.J., HOSSEIN-AZIZUNNESA M.S., BENNET T., NORDLUND K., 2006. A survey to identify economic opportunities for smallholder dairy farms in Bangladesh. Trop. Anim. Health Prod., 38: 131-140.

29. SOMDA J., KAMUANGA M., TOLLENS E., 2005. Characteristics and economic viability of milk production in the smallholder farming systems in The Gambia. Agric. Sys., 85: 42-58.

30. VATIN F., 1996. Le lait et la raison marchande. Essais de sociologie économique. Rennes, France, Presses universitaires, 205 p.

31. VEYRET P., 1951. Géographie de l'élevage. Paris, France, Gallimard, 244 p. (coll. Géographie humaine)

Reçu le 14.05.2007, accepté le 10.04.2008 


\section{Summary}

Morin G., Coulibaly D., Corniaux C., Poccard-Chappuis R., Sidibé S.I., Moulin C.H. Dynamics of Dairy Smallholding Farms Supplying Segou Milk Market in Mali

The increase in fresh milk demand, associated to urban growth, presents an opportunity for developing cattle husbandry around towns. The primary objective of this study was to understand changes in dairy units in the suburban area of Segou, a medium-sized town in Mali, dominated by informal trade. Twenty heads of households were surveyed in an attempt to describe cattle husbandry and milk management in their farms from 1970 to 2005. Three types of dairy units were distinguished: a) mixed farmers with low milk production and milk management by women; b) mixed farmers with sustained milk production and milk management by the heads of households; and c) urban traders with cattle herds for milk production. Urban milk trade was a factor that led to the development of milk production through the extension of the milk collection area (type b), but it did not lead to new production techniques because of the lack of credits (types b and c) or of gender roles in milk management (type a). Nevertheless, cattle holders changed their practices by keeping lactating cows near compounds, increasing mobility for the rest of the herd, administering supplementation during the dry season, and storing crop residues, in response to the decrease in feed availability in rangelands.

Keywords: Dairy cattle - Milk production - Trade - Family Social structure - Mali.

\section{Resumen}

Morin G., Coulibaly D., Corniaux C., Poccard-Chappuis R., Sidibé S.I., Moulin C.H. Dinámicas de las unidades de producción lechera en la cuenca de aprovisionamiento de la ciudad de Ségou (Malí)

El aumento de la demanda de leche fresca, ligado al crecimiento urbano, podría ser una oportunidad para el desarrollo de la cría bovina alrededor de la ciudad. Para la cuenca de aprovisionamiento de la ciudad de Ségou (Malí), dominado por circuitos comerciales informales, el objetivo del estudio fue de comprender las transformaciones de las unidades de producción lecheras. Entre 1970 y 2005, se llevaron a cabo entrevistas con veinte jefes de familia, con el fin de trazar la historia de la cría bovina y la gestión de la leche en sus establecimientos. Se distinguieron tres tipos de unidades lecheras: a) los agro criadores con una producción baja y con una gestión de la leche llevada por las mujeres, b) los agro criadores con una producción lechera estable y con una gestión de leche llevada por el jefe de familia, y c) los comerciantes urbanos con un hato bovino y una orientación lechera. El comercio de la leche en la ciudad fue un factor de desarrollo de la producción lechera, mediante la extensión de la cuenca de aprovisionamiento (unidad tipo b), pero no permitió el surgimiento de nuevas técnicas de producción, particularmente debido a la falta de crédito (tipos b y c) o a la repartición de los roles entre hombres y mujeres en la gestión de la leche (tipo a). Sin embargo, los criadores han hecho evolucionar sus prácticas: distribución de lecherías, aumento de la movilidad del resto del hato, complemento durante la estación seca, almacenamiento de los residuos de los cultivos frente a la escasez de los recursos de pastoreo.

Palabras clave: Ganado de leche - Producción lechera Comercio - Familía - Estructura social - Malí. 